EPJ manuscript No.

(will be inserted by the editor)

\title{
Flow effects on jet profiles and multiplicities
}

\author{
Néstor Armesto ${ }^{\mathrm{a}}$ \\ Department of Physics, CERN, Theory Division, CH-1211 Genève 23, Switzerland \\ Received: date / Revised version: date
}

\begin{abstract}
We study the effects of low- $p_{T}$ collective flow on radiative energy loss from high- $p_{T}$ partons traversing the QCD medium created in high-energy nucleus-nucleus collisions. We illustrate this idea through three examples. Due to longitudinal flow, jet profiles at the LHC present marked asymmetries in the $\eta \times \phi$-plane, and widths in $\eta$ and $\phi$ of particle distributions associated with a high- $p_{T}$ trigger at RHIC become different. Finally, transverse flow implies an increase of high- $p_{T} v_{2}$ at RHIC.
\end{abstract}

PACS. 25.75.Ld Collective flow in relativistic heavy-ion collisions - 24.85.+p Quarks, gluons, and QCD in nuclei and nuclear processes - 25.75.Gz Particle correlations in relativistic heavy-ion collisions

\section{Motivation and formalism}

Low transverse momentum inclusive spectra and azimuthal correlations measured in $\mathrm{Au}+\mathrm{Au}$ collisions at the Relativistic Heavy Ion Collider (RHIC) indicate that different hadron species emerge from a common medium which has built up a strong collective velocity field 1 1, 2, 3. These measurements are broadly consistent with calculations based on ideal hydrodynamics, whose success is regarded as strong evidence [5] that the medium produced in nucleus-nucleus collisions has a very small mean free path, shows a very rapid thermalization at a time less than $1 \mathrm{fm} / \mathrm{c}$ after initial impact, and behaves like an almost ideal fluid with vanishing viscosity. This suggests strong position-momentum correlations in the medium. In this contribution we explore possible effects of this collective flow on high- $p_{T}$ observables 6,7.7.

At collider energies, the production of high- $p_{T}$ hadrons and jets provides a novel independent characterization of the produced medium. This is so since the gluon radiation off parent partons is sensitive to the interaction between the partonic projectile and the medium, see the reviews in 8, 9, 10, 11, 12. The radiative energy loss of a fast parton traversing a QCD medium is determined by momentum exchanges perpendicular to the trajectory of the parton. Thus, if the hard parton (jet) is produced in a frame not co-moving with the collective flow, momentum exchanges become anisotropic and an additional contribution to energy loss comes from flow, see Fig. 11.

At a given energy density $\epsilon$, the dynamic behaviour of the medium is fully specified by its equation of state (EOS)

\footnotetext{
a Present address: Departamento de Física de Partículas and Instituto Galego de Altas Enerxías, Facultade de Física, Campus Sur, Universidade de Santiago de Compostela, 15782 Santiago de Compostela, Spain.
}

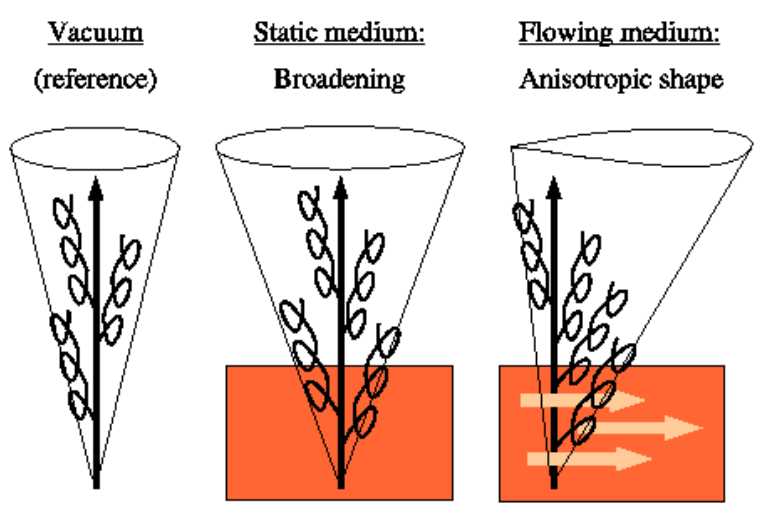

Fig. 1. Sketch of the radiation from a fast parton traversing the vacuum (left), a static medium (center) and a flowing medium (right).

$p=p\left(\epsilon, T, \mu_{B}\right)$ which enters the energy momentum tensor

$$
T^{\mu \nu}(x)=(\epsilon+p) u^{\mu} u^{\nu}-p g^{\mu \nu} .
$$

Here, $u^{\mu}=\gamma(1, \boldsymbol{\beta})$ is the flow velocity field.

On the other hand, quenched high- $p_{T}$ hadroproduction is sensitive to the transport coefficient $\hat{q}$, which is proportional to the density of scattering centres and characterizes the squared average momentum transfer from the medium to the hard parton per unit path length. This transport coefficient is related to $\epsilon$ [13], $\hat{q}\left[\mathrm{GeV}^{2} / \mathrm{fm}\right]=$ $c \epsilon^{3 / 4}\left[\left(\mathrm{GeV} / \mathrm{fm}^{3}\right)^{3 / 4}\right]$. Here, $c$ is a proportionality constant of order unity 13 14.

In order to take into account the effect of the anisotropy in momentum exchanges due to the presence of flow on radiative energy loss, we modify the Yukawa-like scattering 
potential usually employed to model the medium [15,

$$
|a(\mathbf{q})|^{2}=\frac{\mu^{2}}{\pi\left[\left(\mathbf{q}-\mathbf{q}_{0}\right)^{2}+\mu^{2}\right]^{2}} .
$$

The Debye screening mass $\mu$ is usually taken to be proportional to the energy density $\epsilon$, while the directed momentum component $\mathbf{q}_{0}$ is assumed to come from the additional contribution to $T^{i i}$ is given by $\Delta p=(\epsilon+p) u^{i} u^{i}\left(=4 p \gamma^{2} \beta^{2}\right.$ for an ideal $\operatorname{EOS} \epsilon=3 p)$. This implies, for rapidity differences $\eta=0.5,1.0,1.5$ between the frame co-moving with the hard parton and the frame co-moving with the collective flow, $\Delta p / p \simeq 1,5,18$. So this naive estimation shows that such directed component may be $\left|\mathbf{q}_{0}\right| \sim \mu$. Let us indicate that we do not address in this contribution the dynamical dilution of the medium. It can be taken into account by a redefinition of the (time-dependent) transport coefficient as shown in 16 17, 18.

We have calculated the medium-induced radiation of gluons with energy $\omega$ and transverse momentum $\mathbf{k}$, emitted from a highly energetic parton that propagates over a finite path length $L$ in a medium of density $n_{0}$ with collective motion. To first order in opacity, we find 19 20, 21

$$
\begin{aligned}
\omega \frac{d I^{\text {med }}}{d \omega d \mathbf{k}}= & \frac{\alpha_{s}}{(2 \pi)^{2}} \frac{4 C_{R} n_{0}}{\omega} \int d \mathbf{q}|a(\mathbf{q})|^{2} \frac{\mathbf{k} \cdot \mathbf{q}}{\mathbf{k}^{2}} \\
& \times \frac{-L \frac{(\mathbf{k}+\mathbf{q})^{2}}{2 \omega}+\sin \left(L \frac{(\mathbf{k}+\mathbf{q})^{2}}{2 \omega}\right)}{\left[(\mathbf{k}+\mathbf{q})^{2} / 2 \omega\right]^{2}} .
\end{aligned}
$$

In the absence of a medium, the parton fragments according to the vacuum distribution $I^{\text {tot }}=I^{\text {vac }}$. The radiation spectrum (3) characterizes the medium modification of this distribution $\omega \frac{d I^{\mathrm{tot}}}{d \omega d \mathbf{k}}=\omega \frac{d I^{\mathrm{vac}}}{d \omega d \mathbf{k}}+\omega \frac{d I^{\mathrm{med}}}{d \omega d \mathbf{k}}$ in the so-called single hard scattering approximation [19,20,21. Physically equivalent results are obtained in the multiple soft scattering approximation [22], as explained in [21]. From $\omega \frac{d I^{\text {tot }}}{d \omega d \mathbf{k}}$, we calculate distortions of jet energy and jet multiplicity distributions 23 .

\section{Exercises}

To illustrate the effects of flow on high- $p_{T}$ observables and the information about the medium that can be extracted from such measurements, we perform three exercises: We examine jet shapes at the Large Hadron Collider (LHC) 6. 7 in Section 2.1 widths of particle distributions at RHIC 6] in Section 2.2 and, in Section 2.3 high- $p_{T}$ elliptic flow at RHIC [7.

\subsection{LHC: jet shapes}

From $\omega \frac{d I^{\mathrm{tot}}}{d \omega d \mathbf{k}}$, we calculate distortions of jet energy and jet multiplicity distributions 23. Information about $I^{\text {vac }}$ is obtained from the energy fraction of the jet contained in a subcone of radius $R=\sqrt{\eta^{2}+\phi^{2}}, \rho_{\text {vac }}(R)$. For this

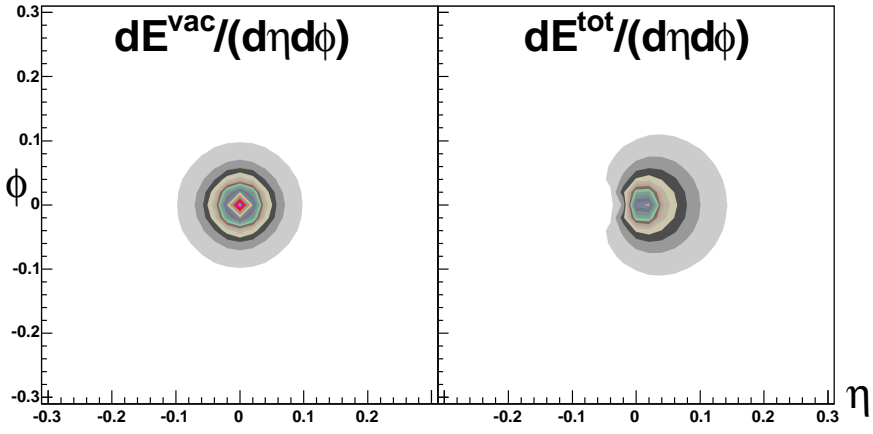

Fig. 2. Calculated distortion of the jet energy distribution in the $\eta \times \phi$-plane for a $100 \mathrm{GeV}$ jet. The right hand-side is for an average medium-induced radiated energy of $23 \mathrm{GeV}$ and equal contributions from density and flow effects, $\mu=q_{0}$.

jet shape, we use the parametrization 24 of the Fermilab $D 0$ Collaboration for jet energies in the range $\approx 50<$ $E_{t}<150 \mathrm{GeV}$ and opening cones $0.1<R<1.0$, modified as explained in 6 7. We then calculate from Eq. (3) the modification [23] of $\rho_{\mathrm{vac}}(R)$ caused by the energy density and collective flow of the medium. To do so, we transform the gluon emission angle $\arcsin (k / \omega)$ in (3) to jet coordinates $\eta, \phi, k d k d \alpha=\omega^{2} \frac{\cos \phi}{\cosh ^{3} \eta} d \eta d \phi$ where $\alpha$ denotes the angle between the transverse gluon momentum $\mathbf{k}$ and the collective flow component $\mathbf{q}_{0}$.

In Fig. 2 we show the medium-modified jet shape for a jet of total energy $E_{T}=100 \mathrm{GeV}$. To test the sensitivity of this energy distribution to collective flow, we have chosen a rather small directed flow component, $q_{0}=\mu$. The parameters in (3) were adjusted [6 7 . such that an average energy $\Delta E_{T}=\int d \omega \omega \frac{d I^{\text {med }}}{d \omega}=23 \mathrm{GeV}$ is redistributed by medium-induced gluon radiation. This is a conservative estimate for $\mathrm{Pb}+\mathrm{Pb}$ collisions at the LHC, and implies a shift of the calorimetric jet center of 0.04 rapidity units [7]. Both the broadening in the jet structure due to mediuminduced Brownian motion of the partonic jet fragments in a dense medium 21] and a marked rotational asymmetry in the $\eta \times \phi$-plane characteristic of the presence of a collective flow field, can be observed. While the jet energy contours shown in Fig. 2 may not be easy to observe, the difference in widths in the different directions in the $\eta \times \phi$-plane should be clearly visible, see [6.7].

\subsection{RHIC: widths of particle distributions}

The calculation of medium-induced gluon radiation is most reliable for calorimetric measurements, but it also provides a framework for the discussion of medium-modified multiplicity distributions 6, 6,23 . At RHIC calorimetric measurements are not currently performed, and the present discussion is on particle distributions associated to high$p_{T}$ triggers [25 26 27]. We have compared our calculation to data of the STAR Collaboration [25 26], which measured the widths of the $\eta$ - and $\phi$-distributions of produced 


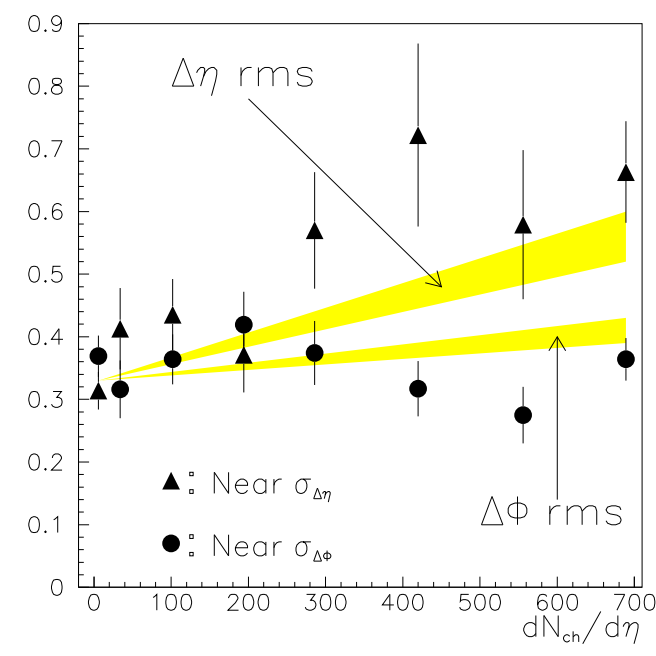

Fig. 3. The width in azimuth and rapidity of the near-side distribution of charged hadrons associated to high- $p_{T}$ trigger particles of transverse momentum $4 \mathrm{GeV}<p_{T}<6 \mathrm{GeV}$ in $\mathrm{Au}+\mathrm{Au}$ collisions at $\sqrt{s_{N N}}=200 \mathrm{GeV}$. Black points are preliminary data from the STAR collaboration [25]. The band represents our calculation for longitudinal flow fields in the range $2<q_{0} / \mu<4$, see text for further details.

hadrons associated to trigger particles of transverse momentum $4 \mathrm{GeV}<p_{T}<6 \mathrm{GeV}$. As a function of centrality of the collision, the $\phi$-distribution does not change within errors, while the $\eta$-distribution shows a significant broadening, see Fig. 3. Although these data are still preliminary, they allow us to illustrate the strategy of determining collective flow effects from jet asymmetries. To this end, we have first used the width of the jet-like correlation in $\mathrm{p}+\mathrm{p}$ collisions to characterize the vacuum contribution. The energy of the parent parton was fixed to $10 \mathrm{GeV}$. We have chosen a rather small in-medium path length $L=2 \mathrm{fm}$ to account for the fact that high- $p_{T}$ trigger particles tend to correspond to parent partons produced near the surface. We then calculated the asymmetry of the broadening in $\Delta \eta$ and $\Delta \phi$ by varying the average momentum transfer between $\mu=0.7$ and $\mu=1.4 \mathrm{GeV}$, and the size of the collective flow component between $q_{0} / \mu=2$ and $q_{0} / \mu=4$. The results thus obtained for central $\mathrm{Au}+\mathrm{Au}$ collisions were extrapolated to peripheral ones by a straight line and are represented by the band in Fig. 3 . Numerical uncertainties in applying calculations of parton energy loss to transverse hadron momenta $p_{T}<10 \mathrm{GeV}$ are significant and have been discussed repeatedly 21. However, the origin of the angular broadening of jet-like particle correlations is essentially kinematic, being determined by the ratio between the momentum transfer from the medium and the energy of the escaping particle; hence, the result in Fig. [3 should not depend strongly on the details of our calculation. We observe that the ratio $q_{0} / \mu=4$, corresponding to a boost of the energy-momentum tensor (1) by approximately one unit in rapidity $\Delta \eta$, can account for the tendency in the preliminary STAR data of Fig. 3 It is consistent with a space-time picture of $\mathrm{Au}+\mathrm{Au}$ collisions at RHIC in which the co-moving frames of the hard parent partons of trigger particles and of the medium are boosted by one rapidity unit.

\subsection{RHIC: elliptic flow}

In general, a hard parton will suffer less energy loss if it propagates on a trajectory parallel to the flow field. Thus, for the same medium-induced suppression, the azimuthal asymmetry at high transverse momentum becomes larger when the contribution of the collective flow field is increased. To estimate the size of this effect, we consider a simple two-dimensional model, see [7] for details. The hard parton is produced at an arbitrary position $\left(x_{0}, y_{0}\right)$ in the transverse plane according to the nuclear overlap. It propagates in its longitudinally co-moving rest frame in the transverse direction $\boldsymbol{n}=(\cos \varphi, \sin \varphi)$, along the trajectory $\mathbf{r}_{\mathbf{0}}(\xi)=\left(x_{0}+\xi \cos \varphi, y_{0}+\xi \sin \varphi\right)$. For simplicity, we assume that the longitudinally co-moving rest frame of this hard parton is the longitudinal rest frame of the medium. Then, there is only a transverse but not a longitudinal flow component. In this exercise we will work in the multiple soft scattering approximation 21,22. For the BDMPS transport coefficient which includes collective flow effects, we make the ansatz

$$
\hat{q}(\xi)=q_{n f}+q_{f}\left|u_{T}\left(\mathbf{r}_{0}(\xi)\right) \cdot \mathbf{n}_{T}\right|^{2} .
$$

Here $q_{f}$ and $q_{n f}$ stand for the flow and non-flow components to $\hat{q}$, the two-dimensional vector $\mathbf{n}_{T}$ is orthogonal to the parton trajectory and projects out the corresponding transverse component of the collective flow field $u_{T}\left(\mathbf{r}_{0}(\xi)\right)$. Defining $q_{n f}$ as the time-averaged transport coefficient of the dynamically equivalent static scenario [16]17,18, the ansatz (4) can account for one of the main effects of longitudinal expansion, namely the time-dependent decrease of the density of scattering centers. In the presence of collective flow, there is an additional momentum transfer orthogonal to the parton trajectory and hence parallel to $\boldsymbol{n}_{T}=(-\sin \varphi, \cos \varphi)$. Since the transport coefficient denotes the squared average momentum transfer per unit path length, this contribution enters quadratically, $\left|u_{T}\left(\mathbf{r}_{0}(\xi)\right) \cdot \mathbf{n}_{T}\right|^{2}$.

For an exploratory model study, we use a blast-wave parameterization of the hadronic freeze-out stage of the collision [28, see 7 for details. With this input, we calculate the characteristic gluon energy and average transverse momentum squared for a parton trajectory in a medium characterized by its density distribution and its collective flow field. With the ansatz (4) for the BDMPS transport coefficient, we find

$$
\begin{aligned}
\omega_{c}\left(\mathbf{r}_{0}, \varphi\right) & =\int_{0}^{\infty} d \xi \xi \hat{q}(\xi) \Omega(\mathbf{r}(\xi), \xi), \\
(\hat{q} L)\left(\mathbf{r}_{0}, \varphi\right) & =\int_{0}^{\infty} d \xi \hat{q}(\xi) \Omega(\mathbf{r}(\xi), \xi) .
\end{aligned}
$$

$\omega_{c}\left(\mathbf{r}_{0}, \varphi\right)$ depends linearly on $q_{n f}$ and on the relative flow strength $q_{f} / q_{n f}$. It increases with increasing flow for parton trajectories which are not parallel to the flow field and 


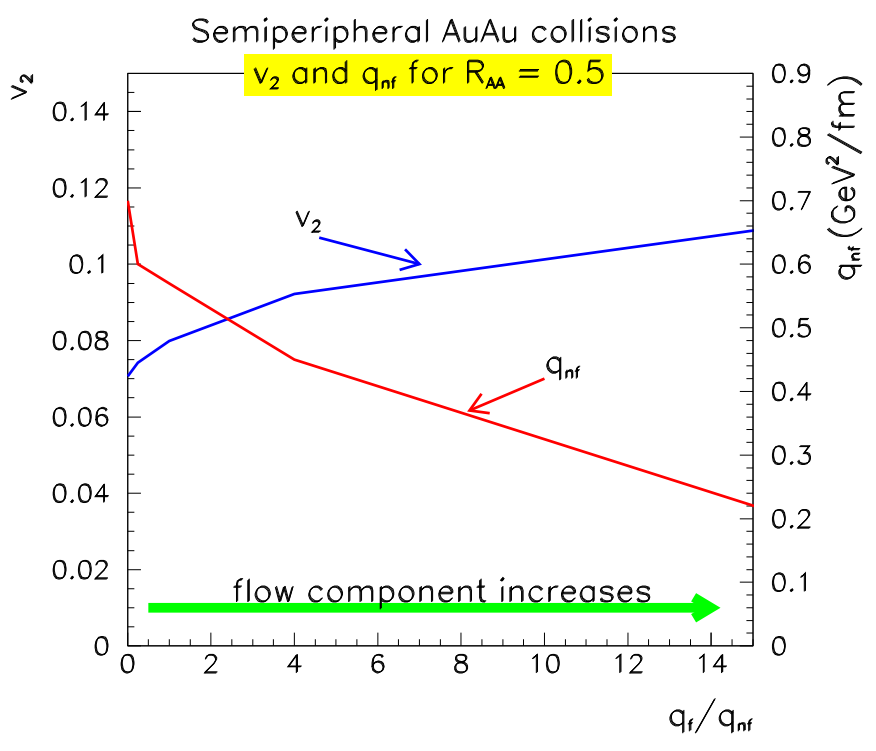

Fig. 4. The dependence of elliptic flow $v_{2}$ and the non-flow component of the BDMPS transport coefficient $q_{n f}$ on the relative flow strength $q_{f} / q_{n f}$, for the case of a nuclear modification factor $R_{A A}=0.5$ in semi-peripheral $\mathrm{Au}+\mathrm{Au}$ collisions for a fixed transverse momentum $p_{T}=7 \mathrm{GeV}$.

shows distortions [7] which, as $\Delta E \approx \alpha_{s} \omega_{c}$ [13], provide a first indication of the extent to which parton energy loss depends on a transverse flow field and affects the azimuthal distribution of inclusive hadron spectra.

To estimate the effects of transverse flow, we get from (5) and (6) the relative suppression of hadronic spectra due to medium-induced parton energy loss $N\left(x_{0}, y_{0}, \varphi, p_{T}\right)$ $=\frac{d \sigma^{m e d}}{d p_{T}} / \frac{d \sigma^{v a c}}{d p_{T}}$. For details of the evaluation, see [7]. The results, see Fig. [4 illustrate two qualitative effects of transverse flow: First, low- $p_{T}$ elliptic flow induces an additional contribution to high- $p_{T}$ azimuthal asymmetry. This effect may reduce significantly the discrepancy of models of parton energy loss 29, 30] in accounting for high- $p_{T} v_{2}$. Second, the presence of collective flow diminishes strongly the local energy density $\epsilon \propto q_{n f}^{4 / 3}$ of the medium required for a nuclear modification factor $R_{A A}$ of fixed size.

\section{Summary}

We have performed an exploratory study of the effects of collective flow on medium-induced radiative energy loss. Jets at LHC 31] may show a clear $\eta-\phi$-asymmetry. At RHIC flow can produce asymmetries in associated particle production for different directions, and an sizable increase of elliptic flow. The determination of densities from jet quenching studies becomes more involved, as flow may mimic energy density. Theoretical uncertainties 12 exist: finite energy corrections, hadronization (which requires pp and $\mathrm{pA}$ data),..., but even a negative result would provide information about the space-time evolution of the system (e.g. of the coupling between hard production and flow).
Truly quantitative answers require the computation of this effect within a full hydrodynamical simulation 32 .

Acknowledgments: The work presented here was made 6.7. in collaboration with Carlos A. Salgado and Urs Achim Wiedemann. I thank R. Baier, P. Jacobs, D. Magestro, A. Morsch, J. Schukraft and F. Wang for useful discussions, and the organizers for such a nice meeting.

\section{References}

1. K. H. Ackermann et al. [STAR Collaboration], Phys. Rev. Lett. 86, (2001) 402.

2. K. Adcox et al. [PHENIX Collaboration], Phys. Rev. Lett. 89, (2002) 212301.

3. S. S. Adler et al. [PHENIX Collaboration], Phys. Rev. Lett. 91, (2003) 182301.

4. P. F. Kolb and U. Heinz, arXiv:nucl-th/0305084

5. M. Gyulassy and L. McLerran, arXiv:nucl-th/0405013

6. N. Armesto, C. A. Salgado and U. A. Wiedemann, Phys. Rev. Lett. 93, (2004) 242301.

7. N. Armesto, C. A. Salgado and U. A. Wiedemann, arXiv:hep-ph/0411341

8. A. Kovner and U. A. Wiedemann, arXiv:hep-ph/0304151

9. M. Gyulassy, I. Vitev, X. N. Wang and B. W. Zhang, arXiv:nucl-th/0302077

10. C. A. Salgado, Mod. Phys. Lett. A 19, (2004) 271.

11. N. Armesto, arXiv:hep-ph/0410162

12. U. A. Wiedemann, these proceedings.

13. R. Baier, Nucl. Phys. A 715, (2003) 209.

14. K. J. Eskola, H. Honkanen, C. A. Salgado and U. A. Wiedemann, Nucl. Phys. A 747, (2005) 511.

15. M. Gyulassy and X. N. Wang, Nucl. Phys. B 420, (1994) 583.

16. R. Baier, Y. L. Dokshitzer, A. H. Mueller and D. Schiff, Phys. Rev. C 58, (1998) 1706.

17. M. Gyulassy, I. Vitev and X. N. Wang, Phys. Rev. Lett. 86, (2001) 2537.

18. C. A. Salgado and U. A. Wiedemann, Phys. Rev. Lett. 89, (2002) 092303.

19. U. A. Wiedemann, Nucl. Phys. B 588, (2000) 303.

20. M. Gyulassy, P. Levai and I. Vitev, Nucl. Phys. B 594, (2001) 371.

21. C. A. Salgado and U. A. Wiedemann, Phys. Rev. D 68, (2003) 014008.

22. R. Baier, Y. L. Dokshitzer, A. H. Mueller, S. Peigne and D. Schiff, Nucl. Phys. B 484, (1997) 265.

23. C. A. Salgado and U. A. Wiedemann, Phys. Rev. Lett. 93, (2004) 042301.

24. B. Abbott, M. Bhattacharjee, D. Elvira, F. Nang and H. Weerts [D0 Coll.], FERMILAB-PUB-97-242-E

25. F. Wang for the STAR Collaboration, talk at QM'04 Conference, Oakland, 11-17 Jan 2004, http://www.lbl.gov/nsd/qm2004/

26. F. Wang [STAR Collaboration], J. Phys. G 30, (2004) S1299.

27. D. Magestro, these proceedings.

28. F. Retiere and M. A. Lisa, Phys. Rev. C 70, (2004) 044907.

29. A. Drees, H. Feng and J. Jia, arXiv:nucl-th/0310044

30. A. Dainese, C. Loizides and G. Paic, Eur. Phys. J. C 38, (2005) 461.

31. A. Accardi et al., arXiv:hep-ph/0310274

32. T. Hirano and Y. Nara, Phys. Rev. C 66, (2002) 041901. 
